\title{
Exact Multifractal Spectra for Arbitrary Laplacian Random Walks
}

\author{
M. B. Hastings \\ Center for Nonlinear Studies and Theoretical Division, Los Alamos National Laboratory, Los Alamos, NM 87545, \\ hastings@cnls.lanl.gov
}

(August 31, 2001)

\begin{abstract}
Iterated conformal mappings are used to obtain exact multifractal spectra of the harmonic measure for arbitrary Laplacian random walks in two dimensions. Separate spectra are found to describe scaling of the growth measure in time, of the measure near the growth tip, and of the measure away from the growth tip. The spectra away from the tip coincide with those of conformally invariant equilibrium systems with arbitrary central charge $c \leq 1$, with $c$ related to the particular walk chosen, while the scaling in time and near the tip cannot be obtained from the equilibrium properties.
\end{abstract}

Diffusion-limited aggregation [1] and the dielectric breakdown model [2](DBM) are central problems in fractal growth which still pose difficult problems. As the growth is controlled by a Laplacian field, the harmonic measure on these clusters is closely connected to the dynamics. Quantitative characterization of the measure is provided by the multifractal exponents [3].

There are unfortunately only a few exact results for these exponents. The Makarov scaling law [4] is true for any simply connected curve, and the electrostatic scaling law [5] is exact for the DBM. In an important recent advance, exact exponents were found for systems that may be described using conformal field theory [6] (CFT); a series of spectra were found, labelled by the central charge $c$ of the theory. This includes both equilibrium statistical mechanics clusters, such as Ising clusters at $c=1 / 2$, as well as certain growth processes, such as random walks at $c=0$.

However, these exponents for growth processes only describe the static properties of these systems. Interesting dynamical questions, such as fluctuations about the average growth rate, remain unanswered in the absence of exact results. To address this, we will consider a nonequilibrium growth process, the Laplacian random walk [6](LRW), and show that its static properties away from the growth tip coincide with those of CFT clusters, but we will also obtain nontrivial scaling near the growth tip, giving rise to nontrivial dynamical scaling. This should shed light on more complicated growth processes such as DLA where the statics and dynamics are so deeply interwoven.

The LRW is defined by a growing walk on a lattice, in which at every stage of growth a Laplacian field is computed with boundary conditions such that it vanishes on the walk and grows logarithmically at infinity. From this field an electric field $E$ is computed, and the probability of selecting any site near the tip for growth is proportional to $E^{\eta}$ at that site, $\eta>0$. This can be considered as a dielectric breakdown model in which growth occurs only at the tip.

In this paper, we propose a model of iterated conformal maps for the LRW. This model is not conformally invariant at the microscopic level, having a fixed parti- cle size; we then show that at large scales this model is equivalent to another discrete model which possesses microscopic conformal invariance. We obtain the exact multifractal spectrum as a continuously varying function of a parameter $m$, which will be indirectly connected to the $\eta$ parameter above. The $\eta=1$ LRW corresponds to $m=1 / 2$ or a $c=-2$ CFT [8]. For other LRWs, there is a continuous relation between $m$ and $c$ for exponents of the measure away from the growth tip. While our model has the same continuum limit as the stochastic Loewner equation [9], we use the discreteness to obtain additional exponents for scaling in time and measure near the growth tip.

Conformal Mappings for the $L R W$ - We use the method of iterated conformal mappings [10] to construct an off-lattice LRW argued to be in the same universality class as that defined above. In the LRW, average growth follows the direction of the electric field at the tip of the walk, but with fluctuations about this preferred direction. We will construct a model with the same features.

Let $F(z)$ be a map from the real line to a growing walk, with $F$ analytic in the upper half plane. Define $f_{x, \lambda}(z)$ to produce a bump of linear size $\sqrt{\lambda}$ at point $x$ on the real line. For example, $f_{0, \lambda}=\sqrt{z^{2}-\lambda}$. If $F(x)$ is the tip of the walk, then $F\left(f_{x, \lambda}(z)\right)$ maps to the walk grown by a distance $\left|F^{\prime}(z)\right| \sqrt{\lambda}$ in the direction of the field at the tip. Thus we propose the following model: fix the growth tip to be the image of $z=0$. At each growth step, first compose $F(z) \rightarrow F\left(f_{0, \lambda}(z)\right)$, to grow the walk, where $\lambda=\left|F^{\prime}(0)\right|^{-2} l_{1}^{2}$ with $l_{1}$ a length that determines the size of the growth step in physical space. Then compose $F(z) \rightarrow F\left(z \pm \sqrt{\lambda} l_{2} / l_{1}\right)$, where the plus or minus sign is chosen randomly. This will shift the growth tip by a distance $l_{2}$ away from the preferred growth direction; the greater the ratio $l_{2} / l_{1}$ the higher will be the dimension of the random walk and so the smaller the effective $\eta$.

We wish to determine the average of the $q$-th moment of $\left|F^{\prime}(z)\right|$ at a point $z=x+i y$; that is, $\left\langle\left|F^{\prime}(z)\right|^{q}\right\rangle$, where the brackets denote ensemble averaging. This describes the field as a function of cutoff in the mathematical plane, $z$, which we we will later convert to scaling in the physical plane. Denote this average after $n$ growth steps as 
$\rho_{q}^{n}(x, y)$. By the chain rule we have

$$
\left|F(f(z))^{\prime}\right|^{q}=\left(1+\frac{\lambda}{2} q \frac{x^{2}-y^{2}}{|z|^{4}}\right)\left|F^{\prime}\left(z-\frac{\lambda}{2 z} \pm \sqrt{\lambda} l_{2} / l_{1}\right)\right|^{q},
$$

where we have used the approximation $f(z)=z-\lambda /(2 z)$, valid at scales larger than $l_{1}$.

Taking a continuum limit in the number of growth steps gives the differential equation

$$
\begin{gathered}
\frac{\partial \rho_{q}(x, y)}{\partial n}=\frac{\lambda}{2}\left(m \partial_{x}^{2}-\frac{x}{x^{2}+y^{2}} \partial_{x}+\frac{y}{x^{2}+y^{2}} \partial_{y}\right. \\
\left.+q \frac{x^{2}-y^{2}}{\left(x^{2}+y^{2}\right)^{2}}\right) \rho_{q}(x, y),
\end{gathered}
$$

where we have replaced the discrete translation $x \rightarrow x \pm$ $\sqrt{\lambda} l_{2} / l_{1}$ by a diffusion term with coefficient $m=\left(l_{2} / l_{1}\right)^{2}$, which defines the "meander" of the walk.

Recall that $\lambda=\left|F^{\prime}(0)\right|^{-2} l_{1}^{2}$. However, by rescaling $\mathrm{d} t=\lambda \mathrm{d} n$ in eq. (2), the dependence of $\lambda$ on $\left|F^{\prime}(0)\right|$ can be removed so that we may assume $\lambda$ is constant. This rescaling does not alter the steady state solution of eq. (2). For growth in radial geometry, the absorber radius of the walk is $e^{t} \equiv F_{1}$, while in a cylindrical geometry, the height of the walk is $t$. Once $\lambda$ is held fixed, eq. (2) is the continuum limit of a growth process in which at each growth step $F(z) \rightarrow F\left(f_{0, s_{1}}\left(z \pm s_{2}\right)\right)$, so that the fixed cutoff in physical space is replaced by a fixed cutoff $s_{1}$ in the mathematical plane. This yields a construction of the growth process as a random combination of conformal maps without memory (all maps chosen independently from one of two possibilities). This dynamics has a microscopic conformal invariance, demonstrating the conformal invariance of the LRW, which could have been anticipated by the known conformal invariance of the $\eta=1$ LRW on-lattice. An image of a walk in cylindrical geometry produced using this microscopically conformally invariant dynamics is shown in Fig. 1, where the density of dots reveals the variation of the physical space cutoff.

The ansatz $\rho_{q}=y^{\gamma_{1}(q)}\left(1+x^{2} / y^{2}\right)^{\gamma_{2}(q)}$ solves eq. (2) with

$$
\begin{gathered}
\gamma_{1}(q)=q-2 m \gamma_{2}(q), \\
\gamma_{2}(q)=\frac{m+1-\sqrt{(m+1)^{2}-2 m q}}{2 m},
\end{gathered}
$$

where we have chosen the sign of the square-root which gives the dominant contribution.

Multifractal Spectrum - From the previous section, $\rho_{q}(0, y)(x, y)$ scales as $y^{\gamma_{1}(q)}$ near the growth tip $(x=$ $0)$, and as $y^{\gamma_{1}(q)-2 \gamma_{2}(q)}$ away from the growth tip. To derive the multifractal spectrum $\tau(q)$ in physical space from these, we define a multifractal spectrum $\tau_{y}(q)$ in the mathematical plane as a preliminary step, and then derive a useful inversion relation between $\tau_{y}(q)$ and $\tau(q)$.
We define $\tau_{y}(q)$ by

$$
\int \frac{\mathrm{d} x}{y}\left|y F^{\prime}(x+i y)\right|^{q} \propto y^{\tau_{y}(q)} .
$$

Define a Legendre transform $f_{y}\left(\alpha_{y}\right)=q \alpha_{y}-\tau_{y}(q)$, with $\alpha_{y}=\tau_{y}^{\prime}(q)$. Then dividing the real line into segments of length $y$, the number of such segments which map under $F$ into points separated by distance $l=y^{\alpha_{y}}$ in the physical plane scales as $y^{-f_{y}\left(\alpha_{y}\right)}$. Conversely, the number of boxes of size $l$ which map, under the inverse of $F$, into size $y=l^{\alpha}$, scales as $l^{-f(\alpha)}$. Equating these yields the inversion relation for $f(\alpha)$

$$
\alpha=1 / \alpha_{y}, \quad f(\alpha)=\alpha f_{y}\left(\alpha_{y}\right) .
$$

From eq. (6) the functional inversion relation for $\tau(q)$ follows,

$$
q=-\tau_{y}(-\tau(q))
$$

We can break the integral in eq. (5) into a contribution away from the growth tip for $x>>y$, and a contribution due to the growth tip for $x \lesssim y$. The first contribution alone would give $\tau_{y}(q)=\gamma_{1}(q)-2 \gamma_{2}(q)+q-1$, while the second alone would give $\tau_{y}(q)=\gamma_{1}(q)+q$. While the true $\tau_{y}(q)$ is obtained by the minimum of these two values, we choose to consider these contributions separately, giving moments away from the tip and near the tip.

The Legendre transform of $\tau_{y}(q)$ away from the tip is $f_{y}\left(\alpha_{y}\right)=1-(1+m)^{2}\left[\left(2-\alpha_{y}\right)+1 /\left(2-\alpha_{y}\right)-2\right] /(2 m)$. This is maximum at $\alpha_{y}=1$ (Makarov's theorem 伯), with $f_{y}(1)=1$ (Gauss's law). The inversion formula (6) gives

$$
f_{\text {away }}(\alpha)=\alpha+\frac{(1+m)^{2}}{4 m}\left(1-\frac{1}{2}\left(2 \alpha-1+\frac{1}{2 \alpha-1}\right)\right) .
$$

This coincides with the CFT spectrum for $c=13-6 m-$ $6 / m$. Thus, $c=1$ implies $m=1$. For self-avoiding walks $(c=0) m=2 / 3$ and for $\eta=1$ on-lattice LRWs $(c=-2)$ $m=1 / 2$. For $m<1$, the fractal dimension $D=1+m / 2$, so the maximum dimension is $3 / 2$.

The Legendre transform of $\tau_{y}(q)$ near the tip is $f_{y}\left(\alpha_{y}\right)=1+m-\left(2-\alpha_{y}\right)(1+m)^{2} /(2 m)-(m / 2)[1 /(2-$ $\left.\alpha_{y}\right)$ ]. In this case, the inversion formula (6) gives

$$
f_{\text {tip }}(\alpha)=-\frac{m}{8(2 \alpha-1)}+\frac{2+m}{4}-\frac{(2+m)^{2}(2 \alpha-1)}{8 m} \text {. }
$$

This has a maximum of zero at $\alpha=(1+m) /(2+m)$. We find the superuniversal result that for $\alpha<2 / 3, f(\alpha)$ is dominated by the tip contribution (9), while for $\alpha>2 / 3$, $f(\alpha)$ is dominated by contributions away from the tip.

Finally, for fixed $y<<x, \rho_{q}(x, y) \propto x^{2 \gamma_{2}}$. This gives a further set of exponents for decay of the field along the cluster as a function of distance $x$ from the tip at small 
$y$, which differ from the exponents for decay of the field at $x=0$ as a function of $y$. In this case a Legendre transform of $2 \gamma_{2}(q)$ yields a probability

$$
x^{-\alpha(m+1)^{2} / 2 m-1 /(2 m \alpha)+(m+1) / m}
$$

of having a field of order $x^{-\alpha}$, with $\alpha>0$. Note that while the most likely value is $\alpha=1 /(m+1)$, it is possible to have fields which are of the same order as the field at the tip. However, there is no scaling relation to connect these exponents to the decay of the field as a function of distance from the tip in physical space at fixed small distance away from the cluster. The reason we had the inversion relations (6) above is that at a distance $y$ from the cluster, the field is smooth on a scale $y$; however, for $y<<x$, the field is not smooth on the scale of $x$.

The exponents (8) are invariant under $m \rightarrow 1 / m$, although the other sets of exponents are not. For $m \geq 1$ the diffusion term is sufficiently strong that the growth process does not give rise to linear walks, but rather clusters, as a point with $y \approx 0$ and large $x$ can diffuse back to $x=0$ despite the advection term in eq. (2) (see Figs. 2,3). While the dimension of such clusters increases above $3 / 2$ as $m$ increases above 1 , the dimension of the perimeter decreases as $m$ increases and remains below $3 / 2$, giving the invariance of exponents away from the tip mentioned above. Unsurprisingly, then, that the exponents near the tip differ and depend on $m$ and not just on $c$.

Dynamical Scaling - There is a form of the electrostatic scaling law [5] for this system. We have (in radial geometry) $\left(\mathrm{d} n / \mathrm{d} F_{1}\right)^{q}=1 /\left(\lambda F_{1}\right)^{q}$. Taking averages on both sides, and noting that $\left\langle\lambda^{-q}\right\rangle \propto$ $\left(F_{1} / l_{1}\right)^{2 q}\left(l_{1} / F_{1}\right)^{\tau(-2 q)+2 q} \propto F_{1}^{-\tau(-2 q)}$, we get

$$
\left\langle\left(\mathrm{d} n / \mathrm{d} F_{1}\right)^{q}\right\rangle \propto F_{1}^{-q-\tau(-2 q)},
$$

where $\tau(q)$ is the scaling near the tip, obtained from Legendre transform of eq. (9). Now consider the average mass, $n$, at given radius, $F_{1}$. Integrating eq. (11), we obtain $\langle n\rangle \propto F_{1}^{-\tau(-2)}$. Since $-\tau(-2)=1+m / 2$, for $m \leq 2$, the dimension obtained from the electrostatic scaling law agrees with that obtained above. This also gives a dimension greater than $3 / 2$ for clusters with $m>1$. However, eq. (11) shows that there are large fluctuations in the growth rate, and that the typical growth is slower than average.

Now we consider the time-averaged growth rate. After transforming to time $t$, eq. (2) has only simple scaling in the mathematical plane, $t \propto \sqrt{y}$. As a first step to finding moments of the time-averaged growth rate, consider the average of a product of $\left|F^{\prime}\right|$ at different times: \langle|$\left.\left.\prod_{i} y_{i} F^{\prime}\left(x=0, y_{i}, t_{i}\right)\right)\left.\right|^{q}\right\rangle$. Suppose all $\left|t_{i}-t_{j}\right|$ are of order $y_{0}^{2}>>y_{i}^{2}$, so that the derivatives are correlated only down to scale $y_{0}$, and uncorrelated below. To determine the effect of the correlations, consider this average for a single time but suppose $F^{\prime}\left(0, y_{0}\right)$ is given, which imposes boundary conditions on eq. (2). Then, $\left\langle\left.\left|\left(\left.y F^{\prime}(0, y)\right|^{q}\right\rangle \propto\left(y / y_{0}\right)^{\tau_{y}(q)}\right| F^{\prime}\left(0, y_{0}\right)\right|^{q}\right.$. Thus, for $r$ points at separate times the ensemble average is

$\left\langle\left|\prod_{i}\left(y_{i} F^{\prime}\left(0, y_{i}, t_{i}\right)\right)\right|^{q}\right\rangle \propto \prod_{i}\left(y_{i} / y_{0}\right)^{\tau_{y}(q)}\left\langle\left|F^{\prime}\left(0, y_{0}\right)\right|^{q r}\right\rangle$.

Transforming to the physical plane we find (given that there is probability $\left(y_{i} / y_{0}\right)^{-f_{y}\left(\alpha_{y}\right)}$ of $y_{i}$ mapping to $l_{i} \propto$ $\left(y_{i} / y_{0}\right)^{\alpha_{y}}\left|F^{\prime}\left(0, y_{0}\right)\right|$ and using arguments as above eq. (6)

$$
\left\langle\left|\prod_{i} l_{i} F^{\prime}\left(l_{i}, t_{i}\right)\right|^{-q}\right\rangle \propto y_{0}^{r q+\tau_{y}(-r \tau(q))} \prod_{i} l_{i}^{\tau(q)} .
$$

We can now obtain moments of the change in mass $\left(n_{2}-n_{1}\right)^{q}$ between times $t_{1}, t_{2}$ by integrating $\int \mathrm{d} n / \mathrm{d} t$ and applying eq. (13).

$$
\left\langle\left(\int_{t_{1}}^{t_{2}} \mathrm{~d} n / \mathrm{d} t\right)^{q}\right\rangle \propto F_{1}^{-q \tau(-2)}\left(t_{2}-t_{1}\right)^{-q+\frac{\tau_{y}(-q \tau(-2))}{2}} .
$$

For $t_{2}-t_{1} \gtrsim 1$, this is $\left\langle\left(\int_{t_{1}}^{t_{2}} \mathrm{~d} n / \mathrm{d} t\right)^{q}\right\rangle \propto F_{1}^{-q \tau(-2)}$, so that the total mass at given radius is self-averaging. Over shorter times, eq. (14) indicates multiscaling of the massradius relation, or dynamical multiscaling connecting the two times $t, n$. The origin of these large fluctuations is that the tip may turn and grow towards the center of the cluster rather than away, so that $F_{1}$ may increase very slowly with mass. The fluctuations in $F_{1}$ are much less in DLA [11], where growth occurs over the whole surface; while $\left|F^{\prime}\right|^{-2}$ may fluctuate locally, the average of $\left|F^{\prime}\right|^{-2}$ over the cluster has much lower fluctuations.

Conclusion and Renormalization Group - We have demonstrated the conformal invariance of the LRW and calculated exactly the multifractal exponents. This provides an opportunity to realize clusters of different conformal field theories as different LRWs, giving an interesting connection between the iterated conformal map technique and CFT. We obtained scaling of harmonic measure both along the curve and near the growth tip. Because we have a solvable dynamic model, we are able to obtain exponents characterizing the scaling in time.

After rescaling of time, the growth process was described by random composition of two different conformal maps. Other fractal sets such as the Julia set are obtained by iterating a single conformal map.

It is interesting to note the lack of universality in these results, so that by changing the ratio $l_{2} / l_{1}$ we are able to obtain continuously varying exponents. In the on-lattice LRW defined above, if successive particles are allowed, instead of attaching to the tip, to attach within some distance from the tip, we expect the dimension to change as the distance is varied, even with $\eta$ held fixed.

Recently, it was argued [12] that for $\eta \geq 4 \mathrm{DBM}$ clusters are asymptotically branchless, and hence onedimensional. One may wonder if instead these branchless 
clusters are LRWs, as suggested previously [13], and have dimension greater than one. However, the lack of universality in the LRW implies that even if such DBM clusters with given $\eta$ were to behave as LRWs, the resulting dimension need not be the same as that of an on-lattice LRW defined with the given $\eta$; this $\eta$ may be renormalized. More importantly, we claim that an asymptotically branchless DBM cannot behave as an LRW; if such a cluster were to behave as an LRW, then the tip would occasionally double back on itself, so that the point of strongest electric field would not be at the tip (as shown, the field at large $x$ in an LRW can be of order the field at the tip), causing a new side branch to appear and contradicting the assumption of no branching. Thus, the possibility of side branching renormalizes the meandering of the LRW to zero and what results is a single straight branch. The asymptotic approach of the dimension to one for DBM clusters with $\eta \geq 4$ is confirmed numerically 14.

Formally, one may extend the RG by noting that the change in growth trajectory induced by a single tipsplitting event gives a logarithmic correction to the cluster size, an additional contribution beyond the correction proportional to branch size considered before [12]. This yields, at lowest order in tip-splitting, a log-squared correction to the cluster size, which is accounted for by an additional scaling field, representing the effective $m$ of the LRW described by the cluster. However, as argued, the possibility of side branching renormalizes the $m$ to zero. Thus, we may ignore these log-squared corrections, as done previously.

Acknowledgements - I thank R. M. Bradley and V. Hakim for motivating this study, and E. Ben-Naim for useful comments. This work was supported by DOE grant W-7405-ENG-36.

[1] T. A. Witten and L. M. Sander, Phys. Rev. Lett. 47, 1400 (1981).

[2] L. Niemeyer, L. Pietronero, and H. J. Wiesmann, Phys. Rev. Lett. 52, 1033 (1984).

[3] T. C. Halsey et. al., Phys. Rev. A 33, 1141 (1986); H. G. E. Hentschel and I. Procaccia, Physica D 8, 435 (1983); T. C. Halsey, K. Honda, and B. Duplantier, J. Stat. Phys. 85, 681 (1996).

[4] N. G. Makarov, Proc. London Math. Soc. 51, 369 (1985).

[5] T. C. Halsey, Phys. Rev. Lett. 59, 2067 (1987).

[6] B. Duplantier, Phys. Rev. Lett. 84, 1363 (2000).

[7] J. W. Lyklema, C. Evertsz, and L. Pietronero, Europhysics Lett. 2, 77 (1986).

[8] S. N. Majumdar, Phys. Rev. Lett. 68, 2329 (1992).

[9] O. Schramm, Israel J. Math. 118 (2000), 221-288; S. Rohde and O. Schramm, math.PR/0106036.

[10] M. B. Hastings and L. S. Levitov, Physica D 116, 244 (1998).
[11] B. Davidovitch et. al., Phys. Rev. E 59, 1368 (1999).

[12] M. B. Hastings, preprint cond-mat/0104344, Phys. Rev. $\mathrm{E}$ in press.

[13] A. Sanchez et. al., Phys. Rev. E 48, 1296 (1993).

[14] M. B. Hastings, preprint cond-mat/0103312, Phys. Rev. Lett. in press.

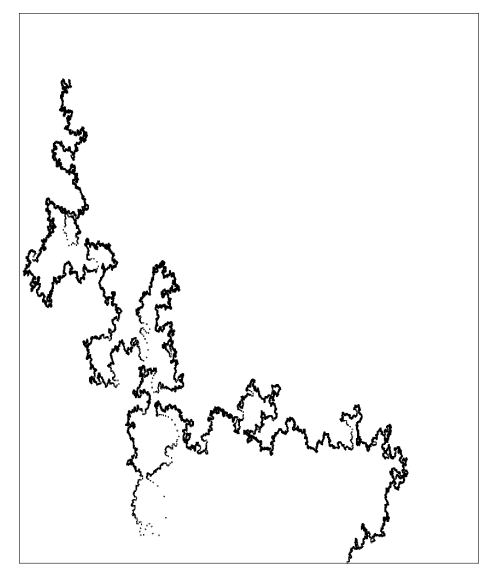

FIG. 1. Plot of an LRW with $m<1$, using conformally invariant microscopic dynamics and $\sim 50,000$ particles. Points indicate position of tip after each growth step.

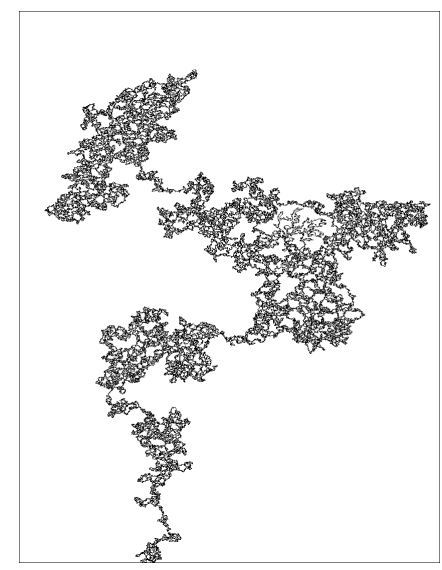

FIG. 2. Plot of an LRW with $m>1$, using fixed cutoff in physical space and $\sim 50,000$ particles. 


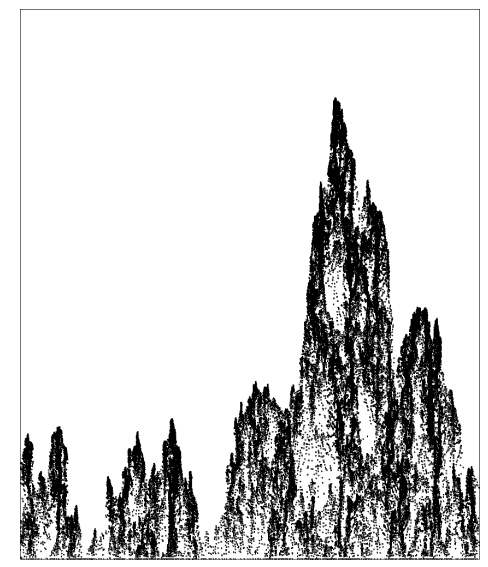

FIG. 3. Plot of an LRW with $m>1$, using conformally invariant microscopic dynamics and $\sim 50,000$ particles. $m$ is greater than for the cluster in Fig. 2. 